\title{
Viscoelastic properties of new mixed wormlike micelles formed by a fatty acid salt and alkylpyridinium surfactant
}

\author{
A. V. Shibaev, O. E. Philippova \\ Department of Physics, Lomonosov Moscow State University, \\ Leninskie Gory, 1, bld. 2, Moscow, 119991, Russia \\ shibaev@polly.phys.msu.ru
}

PACS 36.20.Ey 47.57.Qk 66.20.Ej 82.70.Gg 83.60.Bc

DOI 10.17586/2220-8054-2017-8-6-732-739

\begin{abstract}
We propose a new combination of an anionic (potassium oleate) and cationic (n-octylpyridinium chloride) surfactants that are able to selfassemble into long cylindrical (wormlike) mixed micelles in water. The solutions have strong viscoelastic properties with viscosity up to $300 \mathrm{~Pa} \cdot \mathrm{s}$ and elastic modulus around $20 \mathrm{~Pa}$, which are attributed to the formation of an entangled micellar network. We discover that with an increase in the molar ratio of cationic to anionic surfactant, the solutions first increase drastically their viscosity and elasticity due to the growth of micelles in length and formation of the network, but then the rheological parameters slightly decrease, possibly due to micellar branching or shortening. The addition of cationic surfactant also induces the increase of difference between scission energy and micellar electrostatic energy, which is explained both by stronger binding of surfactants within the micelle and decrease of the micellar net charge.
\end{abstract}

Keywords: surfactant, self-assembly, wormlike micelles, rheology, viscoelastic properties.

Received: 14 November 2017

Revised: 18 November 2017

\section{Introduction}

Long entangled wormlike micelles of surfactants can impart to aqueous solutions pronounced viscoelastic properties [1-15], which are quite sensitive to external stimuli: ionic strength [2,8], type of salt $[11,15]$, temperature [10], various additives including polymers [6,7] and hydrocarbons [12-14]. Such responsiveness is provided by the self-assembled character of micellar chains formed by weak non-covalent interactions [1,9]. Due to responsive viscoelastic properties the wormlike micellar solutions have found use as thickening agents in a wide range of industrial applications including oil recovery [16,17].

Wormlike micelles can be formed by many common ionic surfactants upon addition of salt [2, 8, 11, 15], which shields the repulsion between similarly charged surfactant heads thus making the cylindrical packing more favorable in comparison with spherical one. Since a wormlike micelle consists of a cylindrical central section and semi-spherical end-caps, the increase of the fraction of cylindrical parts at the expense of the spherical ones suggests the growth of wormlike micelles in length.

An alternative approach to obtain wormlike micelles consists in the use of the mixture of two oppositely charged surfactants [3-7]. In this case, the screening of repulsion is even more effective because cationic and anionic surfactant heads are located in close proximity to each other. However, when the composition approaches equimolarity, such mixtures often have a tendency to precipitate [3,5]. To avoid phase separation, it is necessary to take the surfactants differing significantly in the length of hydrophobic tails [4]. At the same time, the tail of one of the surfactants should not be too short, otherwise it will be unable to tune the formation of long micelles [4]. For instance, in the case of sodium oleate, it was demonstrated that its mixture with n-decyl- $\left(\mathrm{C}_{10} \mathrm{TAB}\right)$ or n-dodecyltrimethylammonium bromide $\left(\mathrm{C}_{12} \mathrm{TAB}\right)$ easily phase separates, whereas n-hexyltrimethylammonium bromide $\left(\mathrm{C}_{6} \mathrm{TAB}\right)$ does not induce the formation of long wormlike micelles [4]. Only n-octyltrimethylammonium bromide $\left(\mathrm{C}_{8} \mathrm{TAB}\right)$ gives long micelles without phase separation, even at equimolar ratio [4,6,7]. Thus, the search of an optimally oppositely charged surfactant, which can form long wormlike micelles but avoid precipitation, is a significant challenge.

In the present paper, we propose a new surfactant that induces the formation of long wormlike micelles of potassium oleate and study the rheological properties of the micellar solutions at different surfactant mixture ratios. 


\section{Experimental part}

\subsection{Materials}

Potassium oleate (purity $>98 \%$ ) was purchased from TCI, n-octylpyridinium chloride $\left(\mathrm{C}_{8} \mathrm{Pyr}\right.$, purity $\left.>96 \%\right)$ was obtained from Chemos $\mathrm{GmbH}$, potassium hydroxide (purity $>98 \%$ ) was purchased form Acros. All reagents were used as received. The solutions were prepared in distilled deionized water purified by the Millipore Milli-Q system.

For the preparation of the samples, aqueous stock solutions of potassium oleate and $\mathrm{C}_{8}$ Pyr were first made by dissolving the reagents in water overnight by gentle mixing using a magnetic stirrer. Then, $\mathrm{pH}$ of the solutions was adjusted to $11.0 \pm 0.1$ by adding potassium hydroxide. After that, stock solutions were mixed in appropriate quantities, and the volume was adjusted by adding $10^{-3} \mathrm{M} \mathrm{KOH} \mathrm{(pH} \mathrm{11.0).} \mathrm{The} \mathrm{obtained} \mathrm{samples} \mathrm{were} \mathrm{mixed} \mathrm{by}$ a magnetic stirrer for 1-2 days and left to equilibrate for several days prior to examination.

\subsection{Rheology}

In the rheological experiments, a stress-controlled rotational rheometer Physica MCR 301 (Anton Paar, Austria) was used. The details of the measurements are described elsewhere [6,18]. Cone-plate geometry (diameter $50 \mathrm{~mm}$, cone angle $1^{\circ}$ ) was used for highly viscous samples (with zero-shear viscosity $\eta_{0}>0.1 \mathrm{~Pa} \cdot \mathrm{s}$ ). Double gap coaxial cylinders (mean diameter $26.4 \mathrm{~mm}$, height $40 \mathrm{~mm}$, gap $0.42 \mathrm{~mm}$ ) were used for solutions with lower viscosity $\left(\eta_{0}<0.1 \mathrm{~Pa} \cdot \mathrm{s}\right)$. Temperature was set at $20.00 \pm 0.05{ }^{\circ} \mathrm{C}$ by Peltier elements. A specially constructed measurement cell cover was used to prevent solvent evaporation from the sample during measurement. Prior to investigation, highly viscous and viscoelastic samples were kept in the measurement cell for $10 \mathrm{~min}$ in order to equilibrate them.

In oscillatory shear tests, the angular frequency dependences of the storage $G^{\prime}(\omega)$ and loss $G^{\prime \prime}(\omega)$ moduli were measured. All experiments were performed in the linear viscoelastic regime at the deformation amplitudes $(\gamma)$ of $0.5-5 \%$, at which the storage and loss moduli were independent of deformation amplitude. In order to find the range of amplitudes corresponding to linear viscoelasticity, amplitude sweep tests were first performed, in which the frequency was fixed at $10 \mathrm{rad} / \mathrm{s}$ and the deformation amplitude was varied from $0.01-100 \%$. The plateau storage modulus $G_{0}$ was determined from the $G^{\prime}(\omega)$ curves at the frequency $\omega_{\text {min }}$ where $G^{\prime \prime}$ reaches a minimum. The longest relaxation time $\tau$ was estimated from the frequency $\omega_{0}$ where $G^{\prime}(\omega)$ and $G^{\prime \prime}(\omega)$ intercept, as $\tau=1 / \omega_{0}$.

In steady shear tests, the dependences of viscosity on shear rate (flow curves) were measured. Zero-shear viscosity $\eta_{0}$ of shear-thinning samples was found by fitting the data with the Carreau-Yasuda model, and $\eta_{0}$ for low-viscous samples was taken as a value at a plateau at shear rates $1-201 / \mathrm{s}$, where viscosity is independent of the applied shear rate.

\section{Results and discussion}

In this article, we studied the rheological properties of anionic (potassium oleate) and cationic (n-octylpyridinium chloride or $\mathrm{C}_{8} \mathrm{Pyr}$ ) surfactant mixtures in aqueous solutions. In all experiments, the content of potassium oleate was fixed at $62 \mathrm{mM}(2 \mathrm{wt} \%)$. This concentration is much higher than its critical micelle concentration $(\mathrm{cmc})$, which equals to $\sim 0.9 \mathrm{mM}[19]$, therefore, at this concentration, potassium oleate forms micelles itself in the absence of $\mathrm{C}_{8}$ Pyr. The concentration of $\mathrm{C}_{8} \mathrm{Pyr}$ was varied in the range 0-100 $\mathrm{mM}(0-2.3 \mathrm{wt} \%)$, which corresponds to the molar ratios $\left[\mathrm{C}_{8} \mathrm{Pyr}\right] /$ [potassium oleate] from 0 to 1.6 .

At all the molar ratios under study, the solutions were homogeneous and transparent, and no phase separation was observed. This fact indicates that the attraction force between anionic and cationic surfactant molecules, which arises both from electrostatic attraction of their oppositely charged head groups and hydrophobic interaction between alkyl tails, is not too strong to cause precipitation of catanionic complex. Such a precipitation is inherent for surfactants with lower asymmetry in hydrophobic tail length, e.g. for mixtures of sodium oleate with alkyltrimethylamonium bromides with $\mathrm{C}_{10}$ and $\mathrm{C}_{12}$ tails [4]. In our case, no phase separation is seen even at equimolar composition. According to the literature data, high tail length asymmetry and weaker attraction between anionic and short-chain cationic surfactants leads to the incorporation of cationic species into anionic micelles and to the formation of mixed micelles [4].

The rheological properties of mixed anionic / cationic surfactant solutions were studied at different molar ratios $\left[\mathrm{C}_{8} \mathrm{Pyr}\right] /$ [potassium oleate]. The flow curves are presented in Fig. 1. It is seen that in the absence of $\mathrm{C}_{8} \mathrm{Pyr}$, the viscosity is very low $(0.0012 \mathrm{~Pa} \cdot \mathrm{s})$ and nearly equal to that of water. At these conditions, spherical potassium oleate micelles are formed, as was shown by literature data [20]. Drastic changes in the rheological behavior are seen upon progressive addition of $\mathrm{C}_{8} \mathrm{Pyr}$ (Fig. 1). The viscosity increases by more than 5 orders of magnitude and reaches $300 \mathrm{~Pa} \cdot \mathrm{s}$ for the molar ratio $\left[\mathrm{C}_{8} \mathrm{Pyr}\right.$ ] [potassium oleate] $=0.5$. Also, one can note that at molar ratios 0.4 and higher, the solutions exhibit strong shear-thinning behavior (Fig. 1). 


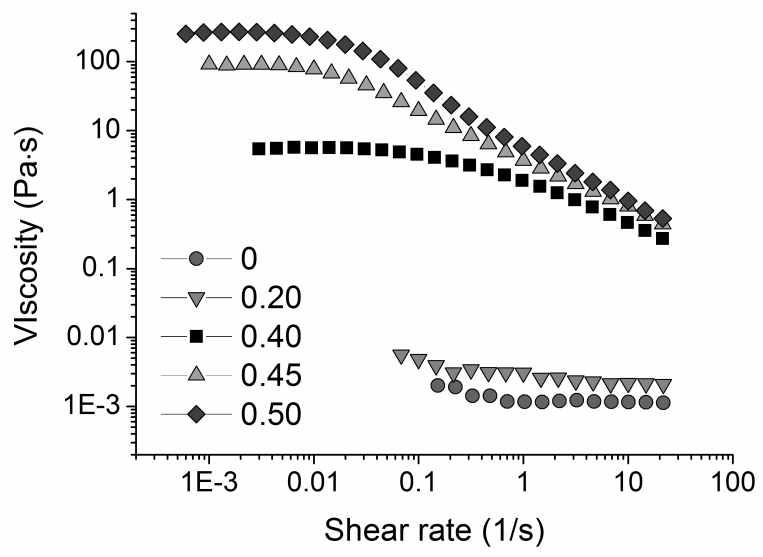

FIG. 1. Flow curves for $62 \mathrm{mM}$ potassium oleate aqueous solutions containing different amounts of $\mathrm{C}_{8} \mathrm{Pyr}$ corresponding to molar ratios $\left[\mathrm{C}_{8} \mathrm{Pyr}\right] /[$ potassium oleate]: 0 (circles), 0.20 (reverse triangles), 0.40 (squares), 0.45 (triangles), 0.50 (diamonds) at $20{ }^{\circ} \mathrm{C}$

The frequency dependences of the storage $\left(G^{\prime}\right)$ and loss $\left(G^{\prime \prime}\right)$ moduli (Fig. 2) show that upon addition of $\mathrm{C}_{8} \mathrm{Pyr}$, the solutions attain strong viscoelastic properties. A well-defined high-frequency elastic plateau appears at $G^{\prime}(\omega)$ curve, whereas the intercept point $\omega_{0}$ between $G^{\prime}(\omega)$ and $G^{\prime \prime}(\omega)$ curves shifts to lower frequencies (by more than two orders of magnitude), indicating the increase of the terminal relaxation time of the system $\tau$, since $\tau=1 / \omega_{0}$. These effects can be explained by the progressive incorporation of $\mathrm{C}_{8}$ Pyr molecules into potassium oleate micelles, resulting in the screening of electrostatic repulsions between the charged oleate head groups, which reduces the average distance between them. This leads to the change of molecular packing parameter of surfactant molecules and to the transformation of spherical micelles into cylindrical ones. When the content of $\mathrm{C}_{8} \mathrm{Pyr}$ is increased, cylindrical (wormlike) micelles grow in length and form an entangled network, thus imparting high viscosity and viscoelastic properties to the solutions.
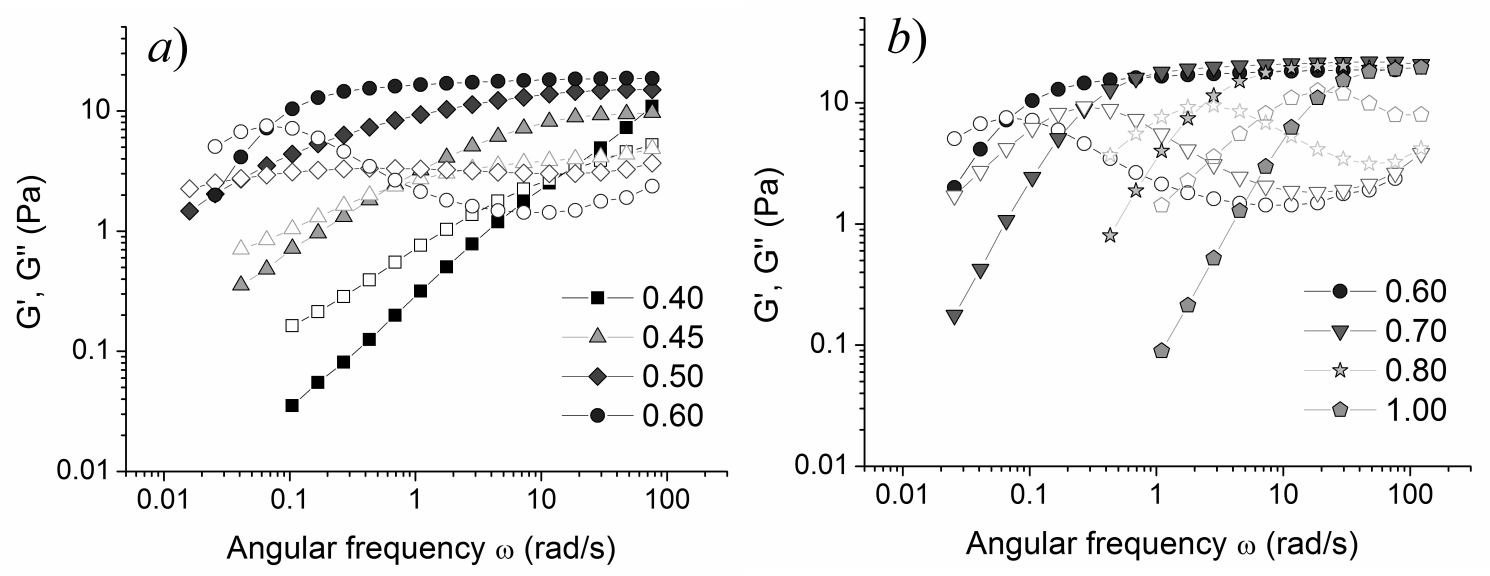

FIG. 2. Frequency dependences of storage $G^{\prime}$ (filled symbols) and loss $G^{\prime \prime}$ (open symbols) moduli for $62 \mathrm{mM}$ potassium oleate aqueous solutions containing different amounts of $\mathrm{C}_{8}$ Pyr corresponding to molar ratios $\left[\mathrm{C}_{8} \mathrm{Pyr}\right] /[$ potassium oleate]: 0.40 (squares), 0.45 (triangles), 0.50 (diamonds), 0.60 (circles), 0.70 (reverse triangles), 0.80 (stars), 1.00 (pentagons) at $20{ }^{\circ} \mathrm{C}$

Figures 3 and 4 show the dependences of the rheological parameters (zero-shear viscosity $\eta_{0}$, relaxation time $\tau$ and plateau storage modulus $G_{0}$ ) on $\mathrm{C}_{8} \mathrm{Pyr}$ concentration. It can be seen that the viscosity and relaxation time first grow, show a maximum around the molar ratio $\left[\mathrm{C}_{8} \mathrm{Pyr}\right] /$ [potassium oleate $]=0.5$, and then decrease. Such behavior was observed for many wormlike surfactant micellar systems [4,8,20,21] including anionic / cationic surfactant mixtures [4]. Initial growth was explained by the increase of the length of linear micelles so that the maximum viscosity corresponds to the formation of longest linear wormlike chains. Further decrease of $\eta_{0}$ and $\tau$ after the maximum is usually attributed to the formation of branched micelles [9]. However, in mixed anionic / cationic 
surfactant systems it may also be due to the shortening of micellar chains as was demonstrated for sodium oleate / $\mathrm{C}_{8} \mathrm{TAB}$ micelles by cryo-transmission electron microscopy [22]. But one should note that the experiments in the last paper [22] were performed at constant total concentration of cationic and anionic surfactants and therefore the amount of long-chain anionic surfactant was decreased as the cationic short-chain surfactant was added, which may favor a decrease of micellar length. In contrast, in the present paper, we keep the anionic surfactant concentration constant. Therefore, one can suppose that for the potassium oleate / $\mathrm{C}_{8}$ Pyr system under study, both branching and shortening of the micelles can be a reason for the decrease of the rheological parameters after the maximum.

As to the elastic modulus $G_{0}$, it grows at molar ratios up to 0.7 and then stays nearly constant (Fig. 4b). The growth, which mainly occurs in the region of steep viscosity increase, can be attributed to the progressive formation of an entangled network of micelles. Indeed, $G_{0}$ is known to be proportional to the number density of entanglements $\rho$ [23]:

$$
G_{0} \sim \rho k T
$$

where $k$ is the Boltzmann constant, and $T$ is the absolute temperature. In wormlike micellar solutions, there is a wide distribution of micellar lengths, and the solutions contain, in particular, very short micelles. At increasing molar ratio $\left[\mathrm{C}_{8} \mathrm{Pyr}\right] /$ [potassium oleate], the short micelles become longer than the entanglement length and start to contribute to $G_{0}$ [24]. At the molar ratio close to 0.7 , the micelles become presumably so long that further change of their size does not influence the number of entanglements in the system.

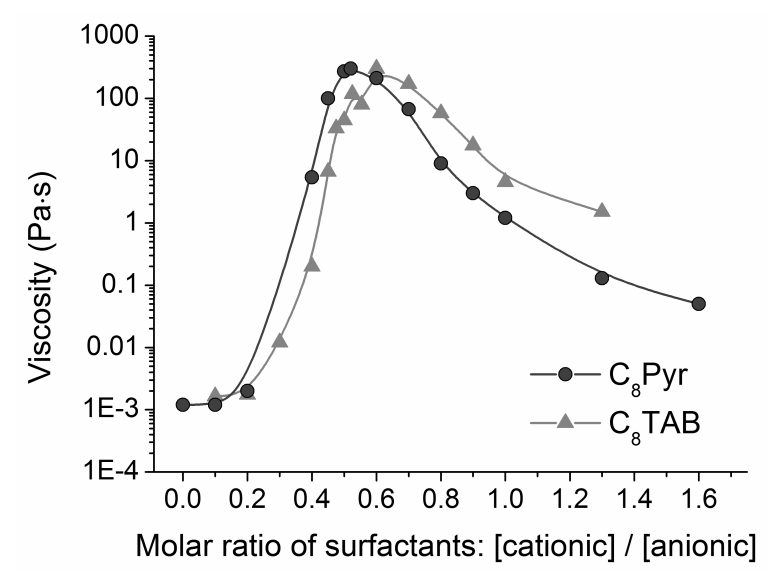

FIG. 3. Dependences of zero-shear viscosity $\eta_{0}$ on the molar ratio [cationic]/[anionic] surfactant for $62 \mathrm{mM}$ aqueous solutions of potassium oleate and different concentrations of $\mathrm{C}_{8} \mathrm{Pyr}$ (circles) or $\mathrm{C}_{8} \mathrm{TAB}$ (triangles) at $20{ }^{\circ} \mathrm{C}$
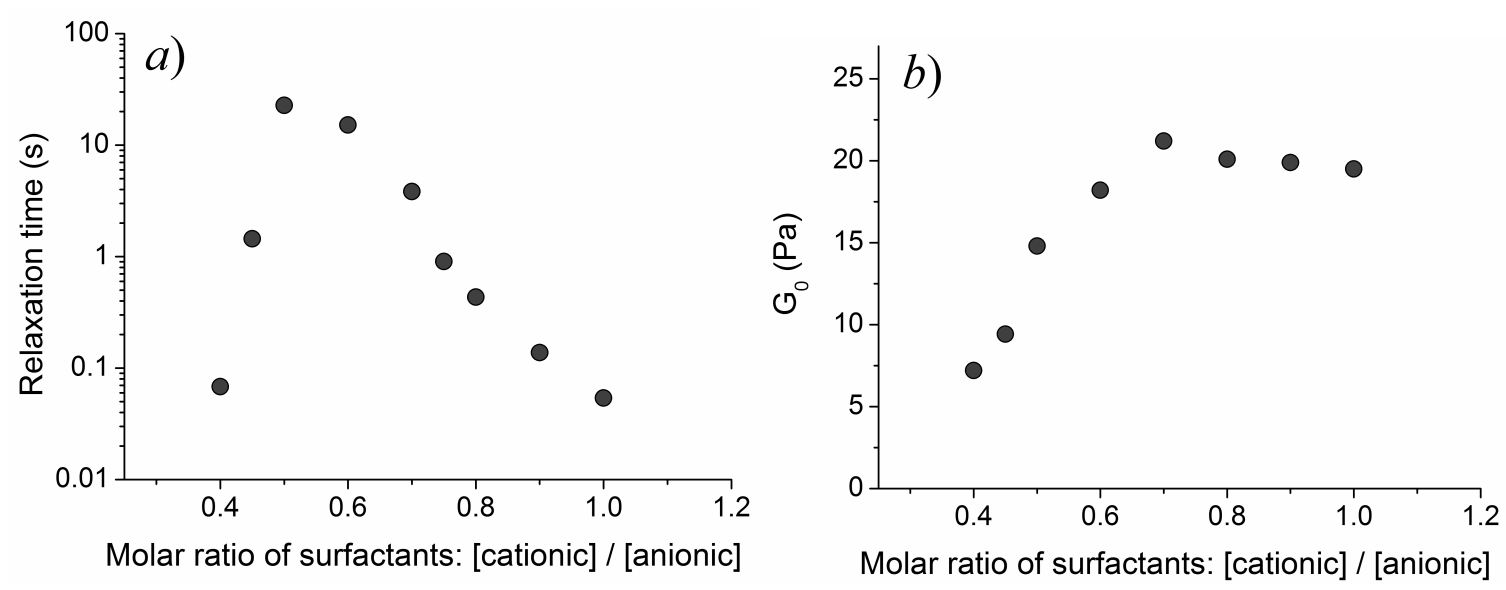

FIG. 4. Dependences of terminal relaxation time $\tau$ (a) and plateau storage modulus $G_{0}$ (b) on the molar ratio [cationic]/[anionic] surfactant for $62 \mathrm{mM}$ aqueous solutions of potassium oleate and different concentrations of $\mathrm{C}_{8} \mathrm{Pyr}$ at $20{ }^{\circ} \mathrm{C}$ 
Figure 3 compares the effect of two different cationic surfactants with the same hydrophobic tail length (C8) but different chemical structure of the polar head on the viscosity of mixed anionic / cationic surfactant solutions. It can be seen that both curves are nearly of the same shape with strongly increasing viscosity at low molar ratios, a maximum, and a slight decrease of viscosity at high cationic surfactant concentrations. This means that the chemical structure of the cationic surfactant polar head has minor influence on the behavior of mixed micelles, which is mostly controlled by the length of its hydrophobic tail [4]. However, it is seen that the maximum for $\mathrm{C}_{8} \mathrm{Pyr}$ is slightly shifted to lower concentrations compared to $\mathrm{C}_{8} \mathrm{TAB}$, and the difference between $\mathrm{C}_{8} \mathrm{Pyr}$ and $\mathrm{C}_{8} \mathrm{TAB}$ grows with their increasing concentration after the maximum. This is probably due to more effective screening of electrostatic repulsions by $\mathrm{C}_{8} \mathrm{Pyr}$ polar head, in which the charge is more accessible than in $\mathrm{C}_{8} \mathrm{TAB}$, where it is sterically shielded by three methyl groups, or by somewhat higher hydrophobicity of $\mathrm{C}_{8}$ Pyr head group in comparison with $\mathrm{C}_{8} \mathrm{TAB}$. Thus, cationic surfactant with pyridinium polar head allows one to obtain solutions with highest viscosity at lower concentration than its trimethylammonium counterpart.

The results obtained show that the molar ratio of cationic to anionic surfactants in wormlike micelles plays a key role in controlling their rheological properties by tuning the micellar length and morphology. All the experiments in this work were performed without added low molecular weight salt, therefore, electrostatic interactions between anionic and cationic surfactant molecules and the amounts of both negative and positive charges are of the major influence on the behavior of the system. Changing the charge ratio (that is, the molar ratio of cationic to anionic surfactants incorporated into the micelles) should affect two main micellar characteristics: 1) the strength of surfactants binding, which determines the micellar scission energy $E_{s c}$ - the energy needed to break a micelle into two shorter micelles, 2) the net charge of micelles, which controls the micellar electrostatic energy $E_{e}$ [9]. When a micelle is cut into two pieces, two hemispherical end-cap need to be formed from the cylindrical part, therefore, the scission energy is equal to the difference between the energy of a cylindrical part and spherical end-cap (containing the same amount of surfactant molecules): $E_{s c}=E_{c y l}-E_{s p h}$. The higher the scission energy, the more energetically favorable are cylindrical parts compared to spherical end-caps, meaning the formation of longer micelles. On the contrary, higher electrostatic energies result in stronger repulsion between the micelles, thus preventing the formation of longer ones. Therefore, the difference between $E_{s c}$ and $E_{e}$ controls the mean length of charged micelles $L$ at low ionic strength [9] via the equation:

$$
L \sim \varphi^{1 / 2} \exp \left(\left(E_{s c}-E_{e}\right) / 2 k T\right)
$$

where $\varphi$ is the volume fraction of the surfactants, $k$ is the Boltzmann constant, and $T$ is the absolute temperature.

The values of $E_{s c}-E_{e}$ for $\mathrm{C}_{8} \mathrm{Pyr} /$ potassium oleate micelles were estimated from the rheological data. Fig. 5a presents the frequency dependences $G^{\prime}(\omega)$ and $G^{\prime \prime}(\omega)$ at different temperatures for the molar ratio $\left[\mathrm{C}_{8} \mathrm{Pyr}\right] /[$ potassium oleate $]=0.6$. From these dependences the values of the plateau modulus $G_{0}$ and the loss modulus at high-frequency minimum $G_{\min }^{\prime \prime}$ were determined, which are related to the average contour length $L$ of micelles by equation [25]:

$$
L / l_{e} \approx G_{0} / G_{m i n}^{\prime \prime}
$$

where $l_{e}$ is the entanglement length. It is argued in the literature that $l_{e}$ is independent of temperature [26,27], therefore, $E_{s c}-E_{e}$ can be determined as a slope of the dependence of $\ln \left(G_{0} / G_{\text {min }}^{\prime \prime}\right)$ on $1 / T$ (Arrhenius plot). These plots for different $\left[\mathrm{C}_{8} \mathrm{Pyr}\right] /$ [potassium oleate] molar ratios are presented in Fig. 6, and the calculated values of $E_{s c}-E_{e}$ are given in Fig. 7 .

From Fig. 7, it is seen that the difference between scission energy and micellar electrostatic energy increases more than twofold when the molar ratio $\left[\mathrm{C}_{8} \mathrm{Pyr}\right.$ / [potassium oleate] is raised from 0.6 to 0.9 . This observation may be explained by two reasons. First, changing the charge ratio between cationic and anionic surfactants may affect the strength of the surfactants' binding. Indeed, incorporation of more cationic species between strongly repelling anionic surfactant polar heads reduces the repulsion between them, thus effectively "binding" them together, which increases the micellar scission energy $E_{s c}$. Second, the increase of cationic to anionic surfactant molar ratio (when it still remains below unity) reduces the net micellar charge thus lowering the micellar electrostatic energy. Namely, $E_{e}$ is proportional to $\nu^{2} \varphi^{1 / 2}$, where $\nu$ is the effective charge per unit length, and $\varphi$ is the total surfactant volume fraction [9]; thus, one can estimate that when the molar ratio $\left[\mathrm{C}_{8} \mathrm{Pyr}\right.$ / [potassium oleate] is increased from 0.6 to $0.9, E_{e}$ drops by approximately 15 times.

So, the length of the micelles is governed both by scission and electrostatic energy, and temperature. When the solutions are heated, the micellar length decreases, exponentially resulting in reduced viscosity. Typical frequency dependences of the complex viscosity modulus $\left|\eta^{*}\right|$ at different temperatures are presented in Fig. 5b for the molar ratio $\left[\mathrm{C}_{8} \mathrm{Pyr}\right] /$ [potassium oleate] $=0.6$. It is seen that the low-frequency plateau (representing the viscosity of the system [28]) lowers at heating. From temperature dependence of viscosity the flow activation energy $E_{a}$ can be 

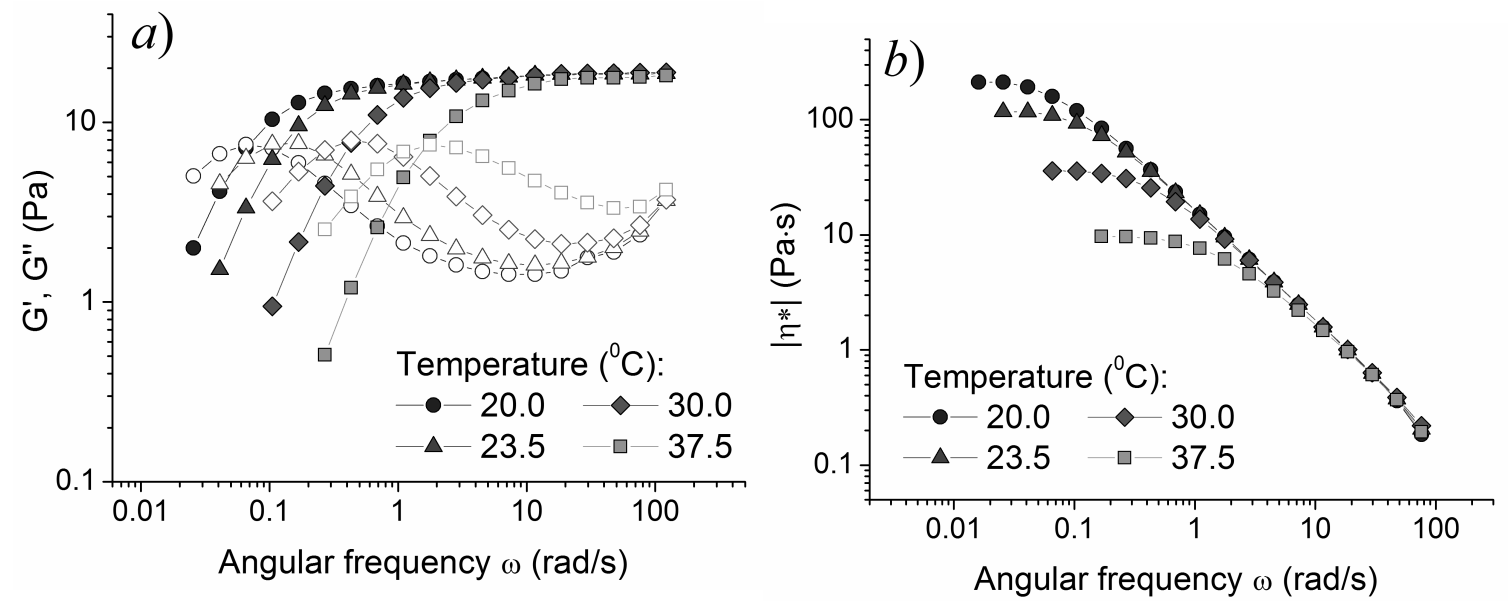

FIG. 5. Frequency dependences of storage $G^{\prime}$ (filled symbols) and loss $G^{\prime \prime}$ (open symbols) moduli (a) and complex viscosity modulus $|\eta *|$ (b) for $62 \mathrm{mM}$ potassium oleate aqueous solutions in the presence of $\mathrm{C}_{8} \mathrm{Pyr}$ (molar ratio $\left[\mathrm{C}_{8} \mathrm{Pyr}\right] /$ [potassium oleate] $=0.6$ ) at different temperatures $\left({ }^{\circ} \mathrm{C}\right): 20$ (circles), 23.5 (triangles), 30 (diamonds), 37.5 (squares)
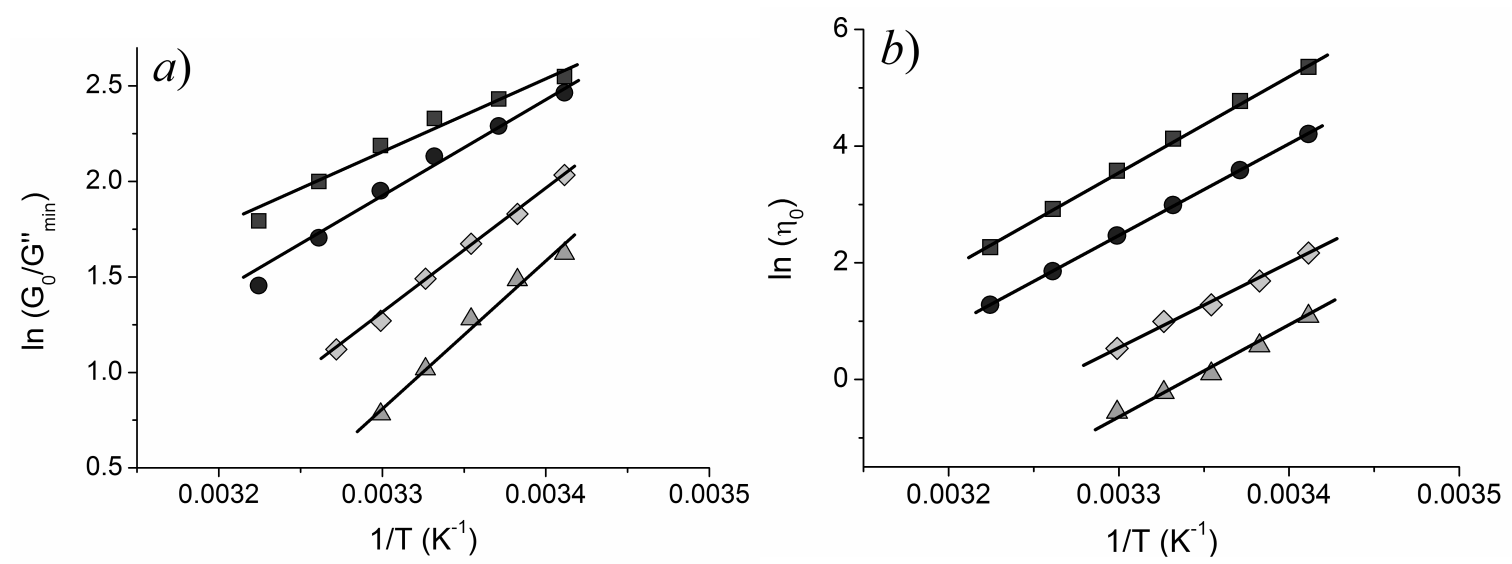

FIG. 6. Dependences of $\ln \left(G_{0} / G_{\text {min }}^{\prime \prime}\right)$ (a) and $\ln \left(\eta_{0}\right)$ (b) on $1 / T$ for $62 \mathrm{mM}$ potassium oleate aqueous solutions in the presence of different amounts of $\mathrm{C}_{8} \mathrm{Pyr}$ corresponding to molar ratios [C $\mathrm{C}_{8} \mathrm{Pyr}$ / [potassium oleate]: 0.6 (squares), 0.7 (circles), 0.8 (diamonds), 0.9 (triangles)

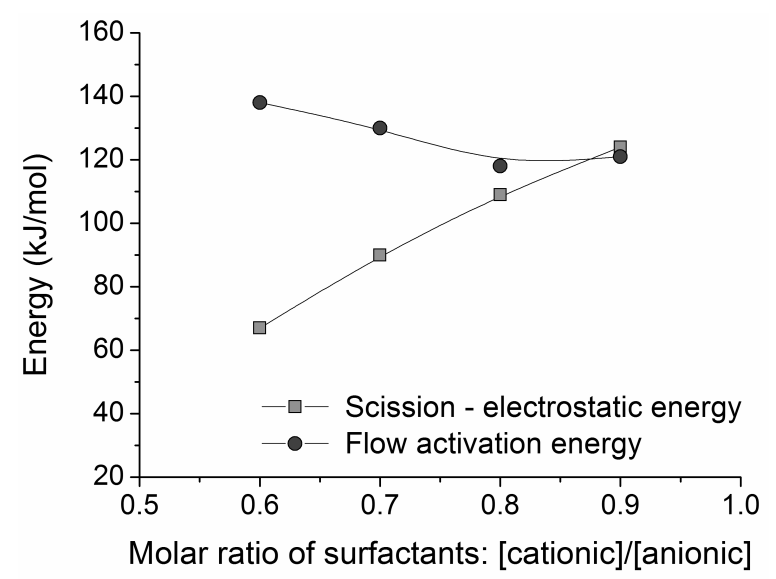

FIG. 7. Dependences of the difference between scission energy and electrostatic energy $E_{s c}-E_{e}$ (a) and flow activation energy $E_{a}$ (b) on the molar ratio [cationic]/[anionic] surfactant for $62 \mathrm{mM}$ aqueous solutions of potassium oleate and different concentrations of $\mathrm{C}_{8} \mathrm{Pyr}$ at $20{ }^{\circ} \mathrm{C}$ 
estimated according to the equation [29]:

$$
\eta \sim \exp \left(E_{a} / k T\right)
$$

The values of $E_{a}$ determined from the Arrhenius plots $\ln \eta$ vs $1 / T$ (Fig. 6b) at different $\mathrm{C}_{8}$ Pyr content are presented in Fig. 7. They are similar to the values reported in literature for different surfactant systems [30,31]. From Fig. 7 it is seen that $E_{a}$ does not significantly change upon variation of $\mathrm{C}_{8} \mathrm{Pyr}$ content indicating that the responsiveness of viscous properties of mixed wormlike micellar solutions to heating is almost unaltered by their composition.

\section{Conclusions}

In this paper, new mixed wormlike micelles consisting of anionic fatty acid salt surfactant and cationic surfactant from the alkylpyridinium family were prepared and investigated. It was discovered that at moderate surfactant concentrations (about $3 \mathrm{wt} \%$ total surfactant) the solutions have high viscosity ( 5 magnitude higher than the viscosity of water) and strong viscoelasticity, which was explained by the formation of an entangled network of wormlike micelles due to incorporation of a short-chain cationic surfactant into anionic micelles and effective screening of electrostatic repulsions on the micellar surface. Temperature measurements at different cationic / anionic surfactant molar ratios revealed that viscoelastic properties of the system are governed both by binding strength of surfactant molecules within the micelle and electrostatic effects. The findings of this paper provide a new way of creating effective surfactant compositions for solutions with highly controllable mechanical properties, with great potential for application, e.g. as thickening agents in the petroleum industry.

\section{Acknowledgments}

This work was supported by the Russian Science Foundation (project No 15-13-00114). The authors express their gratitude to Ms. K. A. Abrashitova (Moscow State University) for help with some rheological measurements.

\section{References}

[1] Rehage H., Hoffmann H. Rheological properties of viscoelastic surfactant systems. J. Phys. Chem., 1988, 92(16), P. 4712-4719.

[2] Khatory A., Lequeux F., Kern F., Candau S.J. Linear and nonlinear viscoelasticity of semidilute solutions of wormlike micelles at high salt content. Langmuir, 1993, 9(6), P. 1456-1464.

[3] Koehler R.D., Raghavan S.R., Kaler E.W. Microstructure and dynamics of wormlike micellar solutions formed by mixing cationic and anionic surfactants. J. Phys. Chem. B, 2000, 104(47), P. 11035-11044.

[4] Raghavan S.R., Fritz G., Kaler E.W. Wormlike micelles formed by synergistic self-assembly in mixtures of anionic and cationic surfactants. Langmuir, 2002, 18(10), P. 3797-3803.

[5] Schubert B.A., Kaler E.W., Wagner N.J. The microstructure and rheology of mixed cationic/anionic wormlike micelles. Langmuir, 2003, 19(10), P. 4079-4089.

[6] Shibaev A.V., Abrashitova K.A., Kuklin A.I., Orekhov A.S., Vasiliev A.V., Iliopoulos I., Philippova O.E. Viscoelastic synergy and microstructure formation in aqueous mixtures of nonionic hydrophilic polymer and charged wormlike surfactant micelles. Macromolecules, 2017, 50(1), P. 339-348.

[7] Shibaev A.V., Kuklin A.I., Philippova O.E. Effect of polymer on the arrangement of mixed anionic/cationic wormlike surfactant micelles revealed by SANS. J. Phys.: Conf. Ser., 2017, 848, P. 012006.

[8] Molchanov V.S., Shashkina Y.A., Philippova O.E., Khokhlov A.R. Viscoelastic properties of aqueous surfactant (potassium oleate) solutions. Colloid J., 2005, 67(5), P. 606-609.

[9] Dreiss C.A. Wormlike micelles: where do we stand? Recent developments, linear rheology and scattering techniques. Soft Matter, 2007, 3(8), P. 956-970.

[10] Chu Z., Dreiss C.A., Feng Y. Smart wormlike micelles. Chem. Soc. Rev., 2013, 42(17), P. 7174-7203.

[11] Koroleva S.V., Victorov A.I. Modeling of the effects of ion specificity on the onset and growth of ionic micelles in a solution of simple salts. Langmuir, 2014, 30(12), P. 3387-3396.

[12] Shibaev A.V., Tamm M.V., Molchanov V.S., Rogachev A.V., Kuklin A.I., Dormidontova E.E., Philippova O.E. How a viscoelastic solution of wormlike micelles transforms into a microemulsion upon absorption of hydrocarbon: new insight. Langmuir, 2014, 30(13), P. 3705-3714.

[13] Shibaev A.V., Molchanov V.S., Philippova O.E. Rheological behavior of oil-swollen wormlike surfactant micelles. J. Phys. Chem. B, 2015, 119(52), P. 15938-15946.

[14] Shibaev A.V., Makarov A.V., Aleshina A.L., Rogachev A.V., Kuklin A.I., Philippova O.E. Structure and oil responsiveness of viscoelastic fluids based on mixed anionic/cationic wormlike surfactant micelles. J. Phys.: Conf. Ser., 2017, 848, P. 012019.

[15] Kwiatkowski A.L., Molchanov V.S., Orekhov A.S., Vasiliev A.L., Philippova O.E. Impact of salt co- and counterions on rheological properties and structure of wormlike micellar solutions. J. Phys. Chem. B, 2016, 120(49), P. 12547-12556.

[16] Yang J. Viscoelastic wormlike micelles and their applications. Curr. Opin. Colloid Interface Sci., 2002, 7(5-6), P. 276-281.

[17] Philippova O.E., Khokhlov A.R. Smart polymers for oil production. Petroleum Chemistry, 2010, 50(4), P. $266-270$.

[18] Philippova O.E., Shibaev A.V., Muravlev D.A., Mityuk D.Yu. Structure and rheology of solutions and gels of stiff polyelectrolyte at high salt concentration. Macromolecules, 2016, 49(16), P. 6031-6040.

[19] Fukuda H., Goto A., Yoshioka H., Goto R., Morigaki K., Walde P. An electron spin resonance study of the pH-induced transformation of micelles to vesicles in an aqueous oleic acid/oleate system. Langmuir, 2001, 17(14), P. 4223-4231. 
[20] Flood C., Dreiss C.A., Croce V., Cosgrove T., Karlsson G. Wormlike micelles mediated by polyelectrolyte. Langmuir, 2005, 21(17), P. 7646-7652.

[21] Raghavan S.R., Kaler E.W. Highly viscoelastic wormlike micellar solutions formed by cationic surfactants with long unsaturated tails. Langmuir, 2001, 17(17), P. 300-306.

[22] Ziserman L., Abezgauz L., Ramon O., Raghavan S.R., Danino D. Origins of the viscosity peak in wormlike micellar solutions. 1. Mixed catanionic surfactants. A cryo-transmission electron microscopy study. Langmuir, 2009, 25(18), P. 10483-10489.

[23] Magid L.J. The surfactant-polyelectrolyte analogy. J. Phys. Chem. B, 1998, 102(21), P. 4064-4074.

[24] Pimenta P., Pashkovski E.E. Rheology of viscoelastic mixed surfactant solutions: effect of scission on nonlinear flow and rheochaos. Langmuir, 2006, 22(9), P. 3980-3987.

[25] Granek R., Cates M.E. Stress relaxation in living polymers: results from a Poisson renewal model. J. Chem. Phys., 1992, 96(6), P. 4758-4767.

[26] Siriwatwechakul W., LaFleur T., Prud'homme R.K., Sullivan P. Effects of organic solvents on the scission energy of rodlike micelles. Langmuir, 2004, 20(21), P. 8970-8974.

[27] Molchanov V.S., Philippova O.E. Dominant role of wormlike micelles in temperature-responsive viscoelastic properties of their mixtures with polymeric chains. J. Colloid Interface Sci., 2013, 394, P. 353-359.

[28] Cox W.P., Merz E.H. Correlation of dynamic and steady flow viscosities. J. Polym. Sci. 1958, 28, P. 619-622.

[29] Fisher P., Rehage H. Rheological master curves of viscoelastic surfactant solutions by varying the solvent viscosity and temperature. Langmuir, 1997, 13(26), P. 7012-7020.

[30] Soltero A., Puig J.E., Manero O. Rheology of the cetyltrimethylammonium tosilate-water system. 2. Linear viscoelastic regime. Langmuir, 1996, 12(11), P. 2654-2662.

[31] Shukla A., Fuchs R., Rehage H. Quasi-anomalous diffusion processes in entangled solutions of wormlike surfactant micelles. Langmuir, 2006, 22(7), P. 3000-3006. 